\title{
Counterparty Credit Exposures for Interest Rate Derivatives using the Stochastic Grid Bundling Method
}

\section{Patrik Karlsson, Shashi Jain \& Cornelis W. Oosterlee}

To cite this article: Patrik Karlsson, Shashi Jain \& Cornelis W. Oosterlee (2016): Counterparty Credit Exposures for Interest Rate Derivatives using the Stochastic Grid Bundling Method, Applied Mathematical Finance

To link to this article: http://dx.doi.org/10.1080/1350486X.2016.1226144

\section{曲 Published online: 02 Sep 2016.}

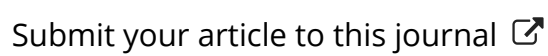

a)

View related articles $\sqsubset$

View Crossmark data $\nearrow$ 


\title{
Counterparty Credit Exposures for Interest Rate Derivatives using the Stochastic Grid Bundling Method
}

\author{
Patrik Karlsson ${ }^{\mathrm{a}, \mathrm{b}}$, Shashi Jain ${ }^{\mathrm{b}}$ and Cornelis W. Oosterlee ${ }^{\mathrm{c}, \mathrm{d}}$ \\ aDepartment of Economics, Lund University, Box 7082, SE-22007 Lund, Sweden; 'buantitative Analytics, \\ ING Bank, Foppingadreef 7, 1000 BV Amsterdam, The Netherlands; 'Centrum Wiskunde and Informatica \\ (CWI), Science Park 123, 1098 XG Amsterdam, The Netherlands; 'Delft Institute of Applied Mathematics, \\ Delft University of Technology, Postbus 5, 2600 AA Delft, The Netherlands
}

\begin{abstract}
The regulatory credit value adjustment (CVA) for an outstanding over-the-counter (OTC) derivative portfolio is computed based on the portfolio exposure over its lifetime. Usually, the future portfolio exposure is approximated using the Monte Carlo simulation, as the portfolio value can be driven by several market risk-factors. For derivatives, such as Bermudan swaptions, that do not have an analytical approximation for their Mark-to-Market (MtM) value, the standard market practice is to use the regression functions from the least squares Monte Carlo method to approximate their MtM along simulated scenarios. However, such approximations have significant bias and noise, resulting in inaccurate CVA charge. In this paper, we extend the Stochastic Grid Bundling Method (SGBM) for the one-factor Gaussian short rate model, to efficiently and accurately compute Expected Exposure, Potential Future exposure and CVA for Bermudan swaptions. A novel contribution of the paper is that it demonstrates how different measures, for instance spot and terminal measure, can simultaneously be employed in the SGBM framework, to significantly reduce the variance and bias of the solution.
\end{abstract}

\section{ARTICLE HISTORY}

Received 5 March 2015

Accepted 12 August 2016

\section{KEYWORDS}

Bermudan Swaptions; credit value adjustment (CVA); Monte Carlo Simulation; stochastic grid bundling method (SGBM); XVA

\section{Introduction}

The notional of outstanding over-the-counter (OTC) derivatives has grown exponentially over the last two decades, a rapid growth mainly due to the increase in interest rate derivatives. Figure 1 illustrates the Bank for International Settlements semiannual market survey of outstanding OTC derivatives from June 1998 through December 2013. As of December 2013, the total amount of outstanding notional in OTC derivatives was 710.2 trillion USD, with 584.4 trillion USD in interest rate derivatives. Any trading desk entering an OTC deal will face the risk that the counterparty at a future date may default and cannot fulfil its payment obligations. Therefore, the bank needs to estimate the total risk it is facing with respect to a particular counterparty and to keep a capital buffer i.e., the capital requirement, to cover for potential losses due to a default. 


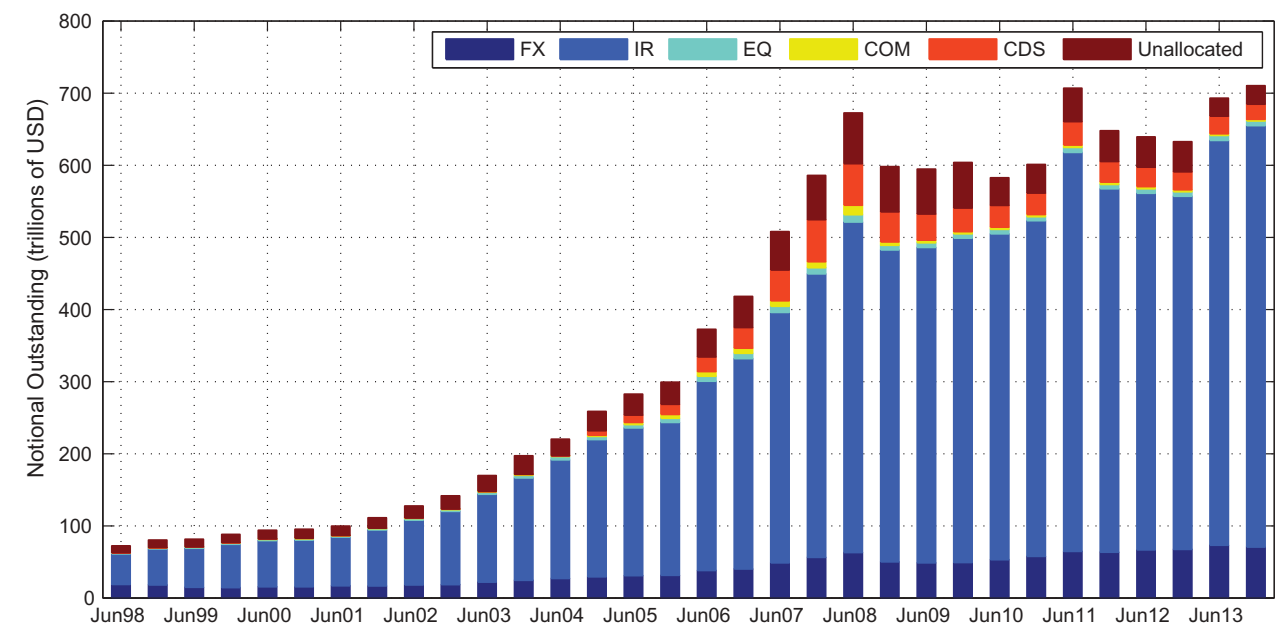

Figure 1. Global OTC derivative markets. The notional amounts (in trillions of US dollars) outstanding of OTC derivatives by risk category from the Bank for International Settlements semiannual survey, June 1998 through December 2013. For, foreign exchange (FX), interest rate (IR), equitylinked (EQ), commodity (COM) derivatives, and credit default swaps (CDS).

Before the financial crisis in 2007, there was the general market view that large companies where 'too-big-to-fail' and thus an overall tendency to underestimate counterparty risk. 'A too-big-to-fail firm is one whose size, complexity, interconnections, and critical functions are such that, should the firm go into liquidation unexpectedly, the financial system and the economy would face severe adverse consequences', to quote the Federal Reserve Chair Ben Bernanke in 2010. However, the bankruptcy of e.g. AIG and Lehman Brothers in 2008 demonstrated that instead of being 'too-big-to-fail' they were instead 'too-big-to-be-allowed-to-fail' (Gregory 2010, 17). These events increased the markets' concern regarding counterparty risk and the need for better risk management when trading OTC derivatives. The Basel Committee on Banking Supervision has formulated in the Basel II and III accords regulatory standards for setting up capital requirements to cover for losses in the case of a counterparty default.

In the Basel II accord, the requirements consist of computing what is generally referred to as the counterparty credit exposure, i.e., the amount of money that can be lost if default occurs, examples of such quantities are the Expected Exposures (EE) and the Potential Future Exposures (PFE). In the Basel III accord, the requirements are more stringent and require the estimation of Credit Valuation Adjustment (CVA) charges (http://www.bis.org/publ/bcbs189_dec2010.pdf). CVA is an adjustment to the derivatives' price to compensate for a possible counterparty default. The value of an OTC deal taking the counterparty risk into account is the value without counterparty risk, the risk-free price, plus a positive adjustment, the CVA charge.

Estimating CVA charges requires an underlying model and, therefore, makes it a model dependent quantity. Products that initially were model independent, for example plain interest rate vanilla swaps become model dependent, because one needs an interest rate model to price the future portfolio exposure at simulated (also model dependent) default times of the counterparty. 
Moving towards exotic derivatives, the situation becomes even more complex since some exotic derivatives are priced using Monte Carlo simulations, and in the context of measuring counterparty risk, EE and PFE are also computed by means of Monte Carlo simulation. Nested Monte Carlo simulations are not an option in this context for performance reasons. Rather than calculating CVA as an over-night job, a trading desk wants to be able to have the CVA estimation for each counterparty in real-time. Also, in order to be able to hedge CVA and restructure portfolios to reduce CVA, there is the challenge of estimating risks and the first-order derivatives for all input parameters.

American Monte Carlo methods, like the well-known Least Squares Method (LSM) as introduced by Longstaff and Schwartz (2001), where the continuation value is approximated by a regression to determine an optimal exercise policy, are today in the context of CVA standard among practitioners, for mainly two reasons. First, because of its ability to increase the computational performance by avoiding nested Monte Carlo methods, i.e., Monte Carlo simulation within a Monte Carlo simulation, by using the same set of paths for pricing and for market simulation, as in De Prisco and Rosen (2005). Secondly, since derivatives such as American and Bermudan swaptions, i.e. products that can be exercised at various dates prior to maturity need to be priced by Monte Carlo methods. The benefit of having an American Monte Carlo CVA calculation is that all instruments will be handled the same way within the CVA computation which makes it easy to aggregate trades, include netting, collateralization, and others.

A general problem with the regression functions as they are used in the least squares Monte Carlo method is that they do not necessarily provide accurate approximations of the MtM value of the derivative over all simulated paths, and can have significant bias and noise, resulting in an inaccurate CVA charge for such products. Additionally, schemes that are used to improve the approximation of the MtM value of such derivatives on the valuation date, for instance using only the in-the-money (ITM) paths for approximation by the regression functions, cannot be used for CVA purposes, as they are based on exposures along all paths and scenarios.

In this paper we extend the Stochastic Grid Bundling Method (SGBM) as introduced by Jain and Oosterlee (2015) for computing future exposure for Bermudan swaptions, where the one-factor Gaussian short rate model is used for simulating the interest rates dynamics. We show through careful numerical experiments, that the EE, PFE and CVA computed using this approach have much smaller errors and noise when compared to using the standard LSM regression based approach. One of the novel contributions of this paper is that under the SGBM problem formulation, in terms of an inner and outer expectation, we can benefit from the flexibility to use different pricing measures within the same computation. Specifically in the case of Bermudan swaptions, this allows us to avoid the simulation of the numeraire process, which helps in achieving significant variance reduction, as compared to the LSM approach. A comprehensive overview of CVA methodologies can be found in Canabarro and Duffie (2003), Picoult (2005), Redon (2006), Pykhtin and Zhu (2007), Pykhtin and Rosen (2010), Gregory (2010) and Brigo and Pallavicini (2013). There is extensive literature on pricing Bermudan swaptions using Monte Carlo schemes, see for instance, Andersen (2000), Bender and Schoenmakers (2006), Kolodko and Schoenmakers (2006) and Piterbarg (2004).

The paper is organized as follows. Section 2 introduces notations, the general framework and formulates the Bermudan swaption pricing. Section 3 describes the 
SGBM algorithm for estimating EE, PFE and CVA charges. In Section 4, we present numerical examples to illustrate the method and its efficiency compared to the traditional LSM. And we conclude in Section 5.

\section{Notation and General Framework}

In this section, we introduce notation, the one-factor Gaussian short rate model (GSR) and define the pricing of Bermudan swaptions. Next, we introduce the methods for estimating counterparty risk using EE, PFE and CVA.

\subsection{The One-Factor Gaussian Short Rate (GSR) Model}

In the general one-factor GSR model the short rate $\boldsymbol{r}(\boldsymbol{t})$ follows a mean-reverting process of the form,

$$
\boldsymbol{d r}(\boldsymbol{t})=\kappa(\boldsymbol{t})(\theta(\boldsymbol{t})-\boldsymbol{r}(\boldsymbol{t})) \boldsymbol{d} \boldsymbol{t}+\sigma(\boldsymbol{t}) \boldsymbol{d W}(\boldsymbol{t}),
$$

where parameter $\kappa(t)$ is the rate of mean-reversion, $\sigma(\boldsymbol{t})$ the volatility, and $\boldsymbol{W}(\boldsymbol{t})$ a standard Brownian motion. The parameters $\kappa(\boldsymbol{t})$ and $\sigma(\boldsymbol{t})$ are usually obtained by calibrating the model to plain-vanilla option prices. The deterministic drift function $\theta(\boldsymbol{t})$ can be directly calculated from the yield curve and fits the curve for

$$
\theta(\boldsymbol{t})=\frac{1}{\kappa(\boldsymbol{t})} \frac{\partial \boldsymbol{f}(0, \boldsymbol{t})}{\partial \boldsymbol{t}}+\boldsymbol{f}(0, \boldsymbol{t})+\frac{1}{\kappa(\boldsymbol{t})} \int_{0}^{t} \boldsymbol{e}^{-2 \int_{u}^{t} \kappa(\boldsymbol{s}) \boldsymbol{d} \boldsymbol{s}} \sigma^{2}(\boldsymbol{u}) \boldsymbol{d u}
$$

A non-smooth initial forward curve can affect the calculation of $\partial \boldsymbol{f}(0, \boldsymbol{t}) / \partial \boldsymbol{t}$, but by defining a new variable $\boldsymbol{x}(\boldsymbol{t})=\boldsymbol{r}(\boldsymbol{t})-\boldsymbol{f}(0, \boldsymbol{t})$, computations are feasible. The dynamics are given by

$$
d x(t)=(y(t)-\kappa(t) x(t)) d t+\sigma(t) d W(t),
$$

where $\boldsymbol{x}(0)=0$ and

$$
y(t)=\int_{0}^{t} e^{-2 \int_{u}^{t} \kappa(s) d s} \sigma^{2}(u) d u
$$

A benefit with the GSR model is that the risk-neutral expectation $\mathbb{E}_{t}^{\mathbb{Q}}[\cdot]$ of the discounted bond price $\boldsymbol{P}(\boldsymbol{t}, \boldsymbol{T})$ at time $t$ with maturity $T$, that is,

$$
P(t, T)=\mathbb{E}_{t}^{\mathbb{Q}}\left[\boldsymbol{e}^{-\int_{t}^{T} r(u) d u}\right],
$$

is known in closed-form and given by

$$
\boldsymbol{P}(\boldsymbol{t}, \boldsymbol{T})=\frac{\boldsymbol{P}(0, \boldsymbol{T})}{\boldsymbol{P}(0, \boldsymbol{t})} \exp \left(-\boldsymbol{x}(\boldsymbol{t}) \boldsymbol{G}(\boldsymbol{t}, \boldsymbol{T})-\frac{1}{2} \boldsymbol{y}(\boldsymbol{t}) \boldsymbol{G}^{2}(\boldsymbol{t}, \boldsymbol{T})\right),
$$




$$
\boldsymbol{G}(\boldsymbol{t}, \boldsymbol{T})=\int_{0}^{t} e^{-\int_{t}^{u} \kappa(s) d s} d u
$$

We use interchangeably the following notations $\mathbb{E}_{t}[\cdot]=\mathbb{E}\left[\cdot \mid \mathcal{F}_{t}\right]$, where $\mathcal{F}_{t}$ is the filtration at time $t$, generated by $\boldsymbol{W}(\boldsymbol{t})$.

The analytic tractability of the GSR model makes it attractive for effective numerical implementations such as for calibration procedures and Monte Carlo simulation, e.g. for pricing and CVA calculations. Criticisms are that the model allows for negative short rates and that it has very limited flexibility for modelling yield curve moments, since all points on the yield curve are perfectly correlated. However, many trading desks today value Bermudan swaptions by using a GSR model, e.g. by the one-factor HullWhite model (HW1F) by Hull and White (1990) due to its simplicity and tractability.

For practical reasons to be explained, we choose to work under the spot measure $\mathbb{Q}^{B}$. The numeraire induced by the spot measure is the discrete version of the continuous compounded money market account with rolling certificate of deposit $\boldsymbol{B}(\boldsymbol{t})$, that is

$$
\boldsymbol{B}(\boldsymbol{t})=\boldsymbol{P}\left(t, T_{i+1}\right) \prod_{n=0}^{i} \boldsymbol{P}^{-1}\left(\boldsymbol{T}_{n}, \boldsymbol{T}_{n+1}\right), t \in\left(\boldsymbol{T}_{i}, \boldsymbol{T}_{\boldsymbol{i}+1}\right],
$$

with corresponding fixed discrete tenor structure, $0=T_{0}<T_{1}<\ldots<T_{N}$. Let $\mathbb{E}_{t}^{B}=\mathbb{E}_{t}$ denote the conditional expectation with respect to the measure induced by $\boldsymbol{B}(\boldsymbol{t})$. One benefit with the spot measure is that the numeraire asset $\boldsymbol{B}(\boldsymbol{t})$ is 'alive' throughout the tenor and, therefore, allows for simulating paths irrespective of the tenor. This is practical for e.g. Bermudan swaptions and American Monte Carlo methods, since the contract can mature randomly at any of the dates in the discrete tenor structure.

Further details on the one-factor Gaussian short rate model, such as derivations of the bond equations, connection to HJM, is out of the scope of this paper, but may be found in Brigo and Mercurio (2001).

\subsection{Bermudan Swaptions}

A vanilla interest rate swap is a contract that allows one to change payments between two different cashflows, often a floating leg against a fixed leg. The values of the forward swap rate $\boldsymbol{S}_{n, m}(\boldsymbol{t})$ and swap annuity $\boldsymbol{A}_{n, m}(\boldsymbol{t})$ at time $t$ with payments $\boldsymbol{T}_{\boldsymbol{n}+1}, \ldots, \boldsymbol{T}_{\boldsymbol{m}}$ are given by.

$$
S_{n, m}(t)=\frac{P\left(t, T_{n}\right)-P\left(t, T_{m}\right)}{A_{n, m}(t)}, A_{n, m}(t)=\sum_{i=n}^{\mathrm{m}-1} \boldsymbol{P}\left(t, \boldsymbol{T}_{i+1}\right) \tau_{i}
$$

where $\tau_{\boldsymbol{i}}=\boldsymbol{T}_{\boldsymbol{i}+1}-\boldsymbol{T}_{\boldsymbol{i}}$. Given a lockout, i.e., a no-call (no-exercise) period up to time $\boldsymbol{T}_{1}$, the Bermudan swaption gives the holder the right - but not the obligation - at a set of fixing dates $\boldsymbol{T}_{\boldsymbol{n}}$, for $\boldsymbol{n} \in \mathcal{I}=\{1,2, \ldots, \boldsymbol{m}-1\}$, i.e., for $\boldsymbol{T}_{\boldsymbol{n}} \in \mathcal{T}=\left\{\boldsymbol{T}_{1}, \boldsymbol{T}_{2}, \ldots, \boldsymbol{T}_{\boldsymbol{m}-1}\right\}$ to enter into a fixed-for-floating swap $\boldsymbol{S}_{\boldsymbol{n}, \boldsymbol{m}}$ with fixing date $\boldsymbol{T}_{\boldsymbol{n}}$ and last payment date $\boldsymbol{T}_{\boldsymbol{m}}$. The Bermudan swaption with the fixed coupon $k$, exercised at time $\boldsymbol{T}_{\boldsymbol{n}}$ corresponds to the payout given by 


$$
U_{n}=\phi \mathcal{N} A_{n, m}\left(T_{n}\right)\left(S_{n, m}\left(T_{n}\right)-k\right)
$$

where $\mathcal{N}$ denotes the notional, and $\phi \in\{-1,+1\}$ the payer or receiver factor $(+1$ for a payer and -1 for a receiver swaption). The holder of a payer Bermudan swaption will pay the fixed swap leg and receive the floating swap leg. The present value $V_{0}$ of a Bermudan swaption is the supremum taken over all discrete stopping times of all conditional expected discounted payoffs, that is,

$$
\boldsymbol{V}_{0}=\boldsymbol{B}\left(\boldsymbol{T}_{0}\right) \sup _{n^{*} \in \mathcal{I}} \mathbb{E}_{T_{0}}\left[\frac{U_{n^{*}}}{\boldsymbol{B}\left(\boldsymbol{T}_{n^{*}}\right)}\right] .
$$

The option value at an arbitrary time $\boldsymbol{T}_{\boldsymbol{n}}$ is the maximum of the intrinsic value $\boldsymbol{U}_{\boldsymbol{n}}$ and the conditional continuation value $\boldsymbol{H}_{n}$, i.e.,

$$
\boldsymbol{V}_{\boldsymbol{n}}=\max \left(\boldsymbol{U}_{\boldsymbol{n}}, \boldsymbol{H}_{\boldsymbol{n}}\right)
$$

where $\boldsymbol{H}_{\boldsymbol{m}}=0$ at maturity $\boldsymbol{T}_{\boldsymbol{m}}$. The continuation value $\boldsymbol{H}_{\boldsymbol{n}}$ is the conditional expected option value at time $\boldsymbol{T}_{\boldsymbol{n}+1}$ and given by,

$$
\boldsymbol{H}_{n}=\boldsymbol{B}\left(\boldsymbol{T}_{n}\right) \mathbb{E}_{T_{n}}\left[\frac{\boldsymbol{V}_{n+1}}{\boldsymbol{B}\left(\boldsymbol{T}_{n+1}\right)}\right] .
$$

The problem is solved via backward induction, starting from the terminal time $\boldsymbol{T}_{\boldsymbol{m}}$, and solved by recursively repeating (4) and (5) until we reach time $\boldsymbol{T}_{0}$, where we get the value $V_{0}$ of the Bermudan swaption contract.

\subsection{Counterparty Credit Risk}

The exposure $\mathrm{E}(\boldsymbol{t})$ towards a counterparty $\mathrm{C}$ at time $t$ is given by the positive side of a contract (or portfolio) value $\boldsymbol{V}(\boldsymbol{t})$, that is,

$$
\mathrm{E}(\boldsymbol{t})=\max \{\boldsymbol{V}(\boldsymbol{t}), 0\} .
$$

This can be seen as the maximum loss if the counterparty defaults at time $t$. Let $\tau_{C}$ denote the counterparty's default time, and the $\mathbb{Q}$-probability that the counterparty $\mathrm{C}$ defaults before time $t$ be given by $\operatorname{PD}(\boldsymbol{t})=\mathbb{Q}\left(\tau_{\boldsymbol{C}}<\boldsymbol{t}\right)$. A commonly used default probability approximation is

$$
\operatorname{PD}(\boldsymbol{t})=1-\exp \left(-\int_{0}^{t} \gamma(\boldsymbol{t}) \boldsymbol{d} \boldsymbol{t}\right),
$$

where the probability factor $\gamma(\boldsymbol{t})$ is called the hazard rate or the instantaneous credit spread, see Gregory (2010). The probability that the counterparty defaults in $d t$ years given that it has not defaulted so far is $\gamma(\boldsymbol{t}) \boldsymbol{d t}$. The default probability for a given counterparty is usually bootstrapped from quoted credit default swaps (CDS). ${ }^{1}$

\subsubsection{Credit Value Adjustment (CVA)}

CVA is the market value of counterparty credit risk, i.e., the difference between the riskfree portfolio value and the value taking into account the counterparty's default probability. The charge is computed as the integral over all points in time of the discounted 
expected exposure given that the counterparty defaults at that time, multiplied with the default probability and the loss given default, i.e., one minus the recovery rate $R$. Following Gregory (2010) the CVA on an instrument (or portfolio) with maturity $T$ is given by

$$
\mathrm{CVA}=(1-\boldsymbol{R}) \boldsymbol{B}(0) \int_{0}^{\boldsymbol{T}} \mathbb{E}\left[\frac{\mathrm{E}(\boldsymbol{t})}{\boldsymbol{B}(\boldsymbol{t})} \delta\left(\boldsymbol{t}-\tau_{C}\right)\right] \boldsymbol{d} \boldsymbol{t}
$$

where $\delta$ is the Dirac delta function, which is one at the counterparty C's default at time $\tau_{C}$, zero otherwise, and $T$ is the maturity of the instrument. Assuming that there is no wrong-way risk (WWR),. ${ }^{2}$ i.e., the default is independent of both the portfolio value and the numeraire, and application of Bayes' theorem, the CVA can be expressed as

$$
\mathrm{CVA}=(1-\boldsymbol{R}) \boldsymbol{B}(0) \int_{0}^{\boldsymbol{T}} \mathbb{E}\left[\frac{\mathrm{E}(\boldsymbol{t})}{\boldsymbol{B}(\boldsymbol{t})} \mid \boldsymbol{t}=\tau_{C}\right] \mathbb{E}\left[\delta\left(\boldsymbol{t}-\tau_{C}\right)\right] \boldsymbol{d} \boldsymbol{t}
$$

The conditional expectation $\mathbb{E}\left[\cdot \mid \boldsymbol{t}=\tau_{\boldsymbol{C}}\right]$ is the current expected exposure at time $t$ given that counterparty $\mathrm{C}$ defaulted at time $t$, i.e., $\boldsymbol{t}=\tau_{C}$. The second expectation within the integrand is the counterparty C's default probability function, i.e., $\boldsymbol{P D}(\boldsymbol{t})$ in (7). The CVA can, therefore, be written as,

$$
\mathrm{CVA}=(1-\boldsymbol{R}) \boldsymbol{B}(0) \int_{0}^{\boldsymbol{T}} \mathbb{E}\left[\frac{\mathrm{E}(\boldsymbol{t})}{\boldsymbol{B}(\boldsymbol{t})} \mid \boldsymbol{t}=\tau_{C}\right] \boldsymbol{d P D}(\boldsymbol{t}) .
$$

Let the expected exposure (EE) and the discounted expected exposure $\operatorname{EE}^{*}(\boldsymbol{t})$ at time $t$ be given by

$$
\begin{gathered}
\mathrm{EE}(\boldsymbol{t})=\mathbb{E}\left[\mathrm{E}(\boldsymbol{t}) \mid \boldsymbol{t}=\tau_{C}\right], \\
\mathrm{EE}^{*}(\boldsymbol{t})=\boldsymbol{B}(0) \mathbb{E}\left[\frac{\mathrm{E}(\boldsymbol{t})}{\boldsymbol{B}(\boldsymbol{t})} \mid \boldsymbol{t}=\tau_{C}\right] .
\end{gathered}
$$

Then, for a discrete time grid $0=\boldsymbol{T}_{0}<\boldsymbol{T}_{1}<\cdots<\boldsymbol{T}_{\boldsymbol{m}}=\boldsymbol{T}$ of observation dates Equation (8) can be approximated by

$$
\mathrm{CVA} \approx(1-\boldsymbol{R}) \sum_{n=1}^{\boldsymbol{m}-1} \mathrm{EE}_{\boldsymbol{n}}^{*}\left(\boldsymbol{P} \boldsymbol{D}_{\boldsymbol{n}+1}-\boldsymbol{P} \boldsymbol{D}_{\boldsymbol{n}}\right)
$$

where $\mathrm{EE}_{n}^{*}=\operatorname{EE}^{*}\left(\boldsymbol{T}_{\boldsymbol{n}}\right)$, to highlight that we work on a discrete time grid.

CVA can be seen as the weighted average of the discounted expected exposure with the weights given by the default probabilities. The complexity of CVA estimation lies within the evaluation of the exposure $\mathrm{E}(\boldsymbol{t})$. Market practice is by American Monte Carlo methods where a large number of market scenarios of factors such as yield and inflation curves, FX rates, equity and commodity prices, credit spreads and others are simulated.

Next to EE and $\mathrm{EE}^{*}$, trading desks are interested in additional exposure profiles such as the PFE. For a given date $t$, the $\alpha$-percentile $\mathrm{PFE}_{\alpha}$ is the maximum exposure of a portfolio with a high degree of statistical confidence $\alpha$ defined as, 


$$
\operatorname{PFE}_{\alpha}(t)=\inf \{x: \mathbb{P}(\mathrm{EE}(t) \leq x) \geq \alpha\}, 0 \leq t \leq T
$$

where $\mathbb{P}$ is the historical probability measure.

\section{Monte Carlo Simulation of Counterparty Credit Risk}

In this section, we summarize the Least Squares Method (LSM) by Longstaff and Schwartz (2001) and present a version of the Stochastic Grid Bundling Method (SGBM) algorithm suitable for CVA calculation of Bermudan swaptions.

There are two choices for estimating the exposures on future scenarios, where the first approach includes all the payments including the one at the observation date, while the second approach only includes the future payments with respect to the observation date. We stay with the latter approach, which in case of cash settled early exercise options implies that the exposure of the option, if not exercised, is equal to its corresponding continuation value along the scenario at time $\boldsymbol{T}_{\boldsymbol{n}}$, i.e., $\boldsymbol{H}_{\boldsymbol{n}}=\boldsymbol{E}_{\boldsymbol{n}}$. If exercised at the observation date, we assume no exposure for the option.

We let market state variable $\boldsymbol{r}_{\boldsymbol{n}}$ represent the simulated market information at time $T_{n}$, and in our case, they are the short rates simulated using the one-factor GSR model in Equation (1).

\subsection{The Least Squares Method (LSM)}

The LSM, introduced by Carriere (1996) and popularized by Longstaff and Schwartz (2001), is a simulation-based method where one approximates the holding value $\boldsymbol{H}_{\boldsymbol{n}}$ at each exercise time $\boldsymbol{T}_{\boldsymbol{n}}$ of a Bermudan option using parametric functions. The parametric functions are approximated using least squares regression, giving the continuation value to have the form,

$$
\boldsymbol{H}_{\boldsymbol{n}}=\sum_{i=0}^{q} \alpha_{i, n} \zeta_{i, n},
$$

for a set of $\boldsymbol{q}$ basis functions $\zeta_{i, n}: \mathbb{R}^{d} \rightarrow \mathbb{R}, \boldsymbol{i}=0,1, \ldots, \boldsymbol{q}$, and regression coefficients $\alpha_{i, n}$. The basis functions $\zeta_{i, n}$ are usually polynomials of the simulated state variables, in our case the short rates, e.g.. $\zeta_{i, n}=r_{n}^{i}$ The regression coefficients are determined, when moving backwards in time, by minimizing

$$
\sum_{\omega \in \Omega_{I T M}\left(\boldsymbol{T}_{n}\right)}\left(\sum_{i=0}^{q} \alpha_{i, n} \zeta_{i, n}(\omega)-U_{\tau_{n}(\omega)}^{*}(\omega)\right)
$$

where $\Omega_{\text {ITM }}\left(\boldsymbol{T}_{\boldsymbol{n}}\right)$ is the subset of paths on which the swaption is in-the-money (ITM) at time $\boldsymbol{T}_{\boldsymbol{n}}$, and

$$
\tau_{\boldsymbol{n}}(\omega)=\min \left(\boldsymbol{T}_{\boldsymbol{j}}: \boldsymbol{U}_{\boldsymbol{j}}(\omega) \geq \boldsymbol{H}_{\boldsymbol{j}}(\omega), \boldsymbol{j}=\boldsymbol{n}+1, \ldots, \boldsymbol{m}\right) .
$$

$\boldsymbol{U}_{n}^{*}(\omega)$ represents the corresponding future cashflows discounted along the path $\omega$, given the observation date $\boldsymbol{T}_{\boldsymbol{n}}$. For the purpose of CVA, we do not make a restriction 
on the paths used for regression (based on whether or not they are ITM). Additionally, to avoid oversight bias, a second set of paths is generated, and regression functions from the initial simulation are used to approximate the continuation value and hence the exposures along the scenarios.

\subsection{The Stochastic Grid Bundling Method (SGBM)}

SGBM is a simulation-based dynamic programming method, which first generates Monte Carlo paths, forward in time (when the diffusion process appears in closed-form or in approximated closed-form the sample paths can be generated directly). This is then followed by finding the optimal early-exercise policy by moving backwards in time. Although SGBM, like LSM, uses least squares regression to approximate parametric functions, the two approaches are significantly different. In loose terms, there are two key differences. First, in the case of LSM, regression is performed on the discounted future cashflows, while in the case of SGBM regression is directly performed on the value function. Second, in LSM, the regressed function is an approximation of the continuation value, and it is used for making the early exercise decisions. In the case of SGBM, the regressed function is an approximation of the option value function in a reduced space. The continuation value for a particular exposure date is determined as the conditional expectation of this regressed functional approximation on the next exposure date. A more detailed description of SGBM can be found in Jain and Oosterlee (2015).

In SGBM, the exposure (continuation value), $\mathrm{E}_{\boldsymbol{n}}$ at time $\boldsymbol{T}_{\boldsymbol{n}}$, is calculated using the law of iterated expectations, that is,

$$
\begin{aligned}
\mathrm{E}_{n} & =\boldsymbol{B}\left(\boldsymbol{T}_{n}\right) \mathbb{E}\left[\frac{\boldsymbol{V}_{n+1}}{\boldsymbol{B}\left(\boldsymbol{T}_{n+1}\right)} \mid \boldsymbol{r}_{n}\right] \\
& =\boldsymbol{B}\left(\boldsymbol{T}_{n}\right) \mathbb{E}\left[\mathbb{E}\left[\frac{\boldsymbol{V}_{n+1}}{\boldsymbol{B}\left(\boldsymbol{T}_{n+1}\right)} \mid \zeta_{n+1}, \boldsymbol{r}_{n}\right] \mid \boldsymbol{r}_{\boldsymbol{n}}\right],
\end{aligned}
$$

where $\zeta_{\boldsymbol{n}}=\left(\zeta_{0, \boldsymbol{n}}, \ldots, \zeta_{\boldsymbol{q}, \boldsymbol{n}}\right)^{\mathrm{T}}$ is a $q$-dimensional set of basis functions. For Bermudan swaptions in the one-factor Gaussian short rate model, we take a polynomial of the short rates as the basis functions. Writing the expected exposure as in Equation (11), decomposes the problem into two steps. The first step involves computing the inner conditional expectation,

$$
\boldsymbol{Z}_{n+1}=\mathbb{E}\left[\frac{\boldsymbol{V}_{n+1}}{\boldsymbol{B}\left(\boldsymbol{T}_{n+1}\right)} \mid \zeta_{n+1}, \boldsymbol{r}_{n}\right],
$$

which is followed by the computation of the outer expectation,

$$
\mathrm{E}_{n}=\boldsymbol{B}\left(\boldsymbol{T}_{n}\right) \mathbb{E}\left[\boldsymbol{Z}_{n+1} \mid r_{n}\right]
$$

By carefully selecting the basis functions, Equation (13) can be computed in 'closedform'. However, numerical approximations are required to calculate $Z_{n+1}$ in Equation (12).

In order to compute $\boldsymbol{Z}_{\boldsymbol{n}+1}$ in Equation (12), $\boldsymbol{V}_{\boldsymbol{n}+1}$ needs to be conditioned on $\boldsymbol{r}_{\boldsymbol{n}}$. If computational costs were not a concern, this would imply simulating a new set of 
scenarios originating from each $\boldsymbol{r}_{\boldsymbol{n}}(\omega)$ and projecting the corresponding $\boldsymbol{V}_{\boldsymbol{n}+1}$ for these sub-scenarios onto the basis functions $\zeta_{n+1}$. This would result in a regressed function for each outer scenario $\boldsymbol{r}_{\boldsymbol{n}}(\omega)$ at $\boldsymbol{T}_{\boldsymbol{n}}$. However, nested Monte Carlo simulation is computationally inefficient as the number of paths grows exponentially with each time step.

A practical approach to condition $\boldsymbol{V}_{\boldsymbol{n}+1}$ on $\boldsymbol{r}_{\boldsymbol{n}}$ is to use bundling techniques. Bundling was introduced by Tilley (1993) and extended to higher dimensions in the State Space Partitioning Method (SSPM) in Jin, Tana, and Sun (2007), and is a method to partition the state space into non-overlapping regions, so that each point in the space can be identified to lie in exactly one of the bundled regions. The intuitive idea behind bundling is that for $\boldsymbol{r}_{\boldsymbol{n}}(\omega)$, if the neighbouring paths are grouped together, the resulting distribution of paths at the next time step, in the limiting case of infinite scenarios and bundles, would be similar to the one obtained if new scenarios were generated starting from $\boldsymbol{r}_{\boldsymbol{n}}(\omega)$.

At each time $\boldsymbol{T}_{n}$, the paths $\boldsymbol{r}_{\boldsymbol{n}}\left(\omega_{k}\right)$, for $\boldsymbol{k}=1, \ldots, \boldsymbol{K}$, are clustered into sets of nonoverlapping bundles $\mathcal{B}\left(\boldsymbol{T}_{\boldsymbol{n}}\right)$. We bundle the grid points at each time step using the recursive bifurcation algorithm, explained in Appendix B. The number of bundles, after $p$ iterations, equals $2^{p}$. Figure 2 illustrates the bundling procedure with 2 and 4 bundles. The computational complexity for the bundling is linear in the total number of grid points $K$, the dimensions $d$, and the number of iteration steps $p$. This makes the method of bundling practical and fast.

The inner expectation, $\boldsymbol{Z}_{\boldsymbol{n}+1}$ given by Equation (12) is then approximated onto a polynomial subspace where the values are linear combinations of the basis functions. This is done by regressing locally, within each bundle, the option values, divided by the corresponding bank account process, at $\boldsymbol{T}_{\boldsymbol{n}+1}$ for those paths that originate from the $s$-th bundle which contains $\boldsymbol{r}_{\boldsymbol{n}}\left(\omega_{\boldsymbol{k}}\right)$, that is

$$
\widehat{\boldsymbol{Z}}_{n+1}^{s}=\sum_{i=0}^{q} \alpha_{i, n+1}^{s} r_{n+1}^{i}, \quad r_{n}\left(\omega_{k}\right) \in \mathcal{B}_{n}^{s}
$$

such that the following residual is minimized
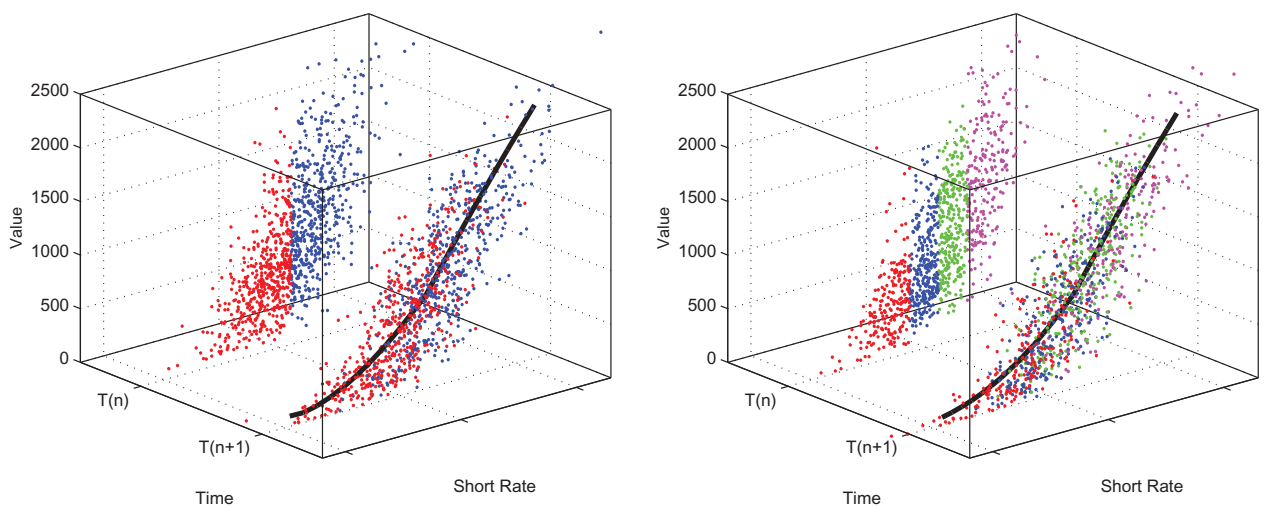

Figure 2. Continuation values approximated by a third-order polynomial, with the short rates as basis functions. Left: Regression with 2 bundles. Right: Regression with 4 bundles. 


$$
\min _{\alpha^{s}} \sum_{\boldsymbol{r}_{n}(\omega) \in \mathcal{B}_{n}^{s}}\left(\widehat{\boldsymbol{Z}}_{\boldsymbol{n}+1}^{s}(\omega)-\frac{\boldsymbol{V}_{\boldsymbol{n}+1}(\omega)}{\boldsymbol{B}\left(\boldsymbol{T}_{\boldsymbol{n}+1}, \omega\right)}\right)^{2}
$$

The exposure at a grid point $\boldsymbol{r}_{\boldsymbol{n}}\left(\omega_{\boldsymbol{k}}\right)$ that belongs to bundle $\mathcal{B}_{\boldsymbol{n}}^{\boldsymbol{s}}$ is then approximated by,

$$
\begin{aligned}
\widehat{\mathrm{E}}_{\boldsymbol{n}}\left(\omega_{\boldsymbol{k}}\right) & =\boldsymbol{B}\left(\boldsymbol{T}_{\boldsymbol{n}}, \omega_{\boldsymbol{k}}\right) \mathbb{E}\left[\widehat{\boldsymbol{Z}}_{\boldsymbol{n}+\boldsymbol{1}}^{\boldsymbol{s}} \mid \boldsymbol{r}_{\boldsymbol{n}}\left(\omega_{\boldsymbol{k}}\right)\right] \\
& \approx B\left(T_{n}, \omega_{k}\right) \sum_{i=0}^{q} \alpha_{i, n+1}^{s} \mathbb{E}\left[\zeta_{i, n+1} \mid r_{n}\left(\omega_{k}\right)\right] .
\end{aligned}
$$

Equation (16) converges to the true expected exposure, when the number of asset paths $K$ and $i$ the number of bundles tend to infinity, see Jain and Oosterlee (2015) for details.

Once we have calculated exposures at each time step $\boldsymbol{T}_{n}$ using Equation (16) we can approximate the expected exposure as

$$
\widehat{\mathrm{EE}}_{n} \approx \frac{1}{K} \sum_{k=1}^{K} \widehat{\mathrm{E}}_{n}\left(\omega_{k}\right)
$$

and the discounted expected exposure as

$$
\widehat{\mathrm{EE}}_{n}^{*} \approx \frac{1}{\boldsymbol{K}} \sum_{\boldsymbol{k}=1}^{\boldsymbol{K}} \boldsymbol{B}\left(\boldsymbol{T}_{0}\right) \frac{\widehat{\mathrm{E}}_{\boldsymbol{n}}\left(\omega_{\boldsymbol{k}}\right)}{\boldsymbol{B}\left(\boldsymbol{T}_{\boldsymbol{n}}, \omega_{\boldsymbol{k}}\right)}
$$

for $\boldsymbol{k}=1, \ldots, \boldsymbol{K}$. Then, by using Equation (9), the CVA charges follow.

Regression-based American Monte Carlo methods depend on the choice of the regression variables. To avoid over-fitting one should not use too many regression variables since they are easily affected by outliers in the simulation. For Bermudan swaptions it is common to use a second-order polynomial (of the underlying swap value or the short rate) for the regression, see for instance, Glasserman and Yu (2004).

Remark 3.1 As the regression approximation depends on a rather arbitrary choice of the basis functions, one should ideally have an estimate of both the upper and lower bound values for the true price. A lower bound for the option price can be computed using the so-called path-estimator approach, where the option value is computed as an expectation of the discounted payoffs from a sub-optimal exercise policy, see for example, Broadie and Glasserman (2004). The policy is sub-optimal, because of the numerical errors in its computation. One should use a fresh set of paths for the path-estimator, and not the same ones used to obtain the early-exercise policy, to avoid a foresight bias. An upper bound is found using the duality approach, based upon the work of Rogers (2002) and Haugh and Kogan (2004). This approach has moreover been extensively studied and described in Andersen \& Broadie (2004) and Kolodko and Schoenmakers (2004). Belomestny, Bender, and Schoenmakers (2009) present an efficient method for obtaining the upper bound using the duality approach, which can be used for Bermudan swaptions. The 
quality of the upper bound produced by the duality approach depends on the quality of the estimated exercise policy in the first pass, a more accurate policy gives tighter upper and lower bounds. In this paper, however, we focus only on the lower bounds and show that the ones obtained using SGBM, with significantly fewer paths, converge to the same lower bound value as those obtained using LSM. One can, in a relatively straightforward way, use the exercise policy obtained from SGBM in the duality approach to obtain a corresponding upper bound.

\subsubsection{Hybrid Measure Monte Carlo}

CVA calculations are done at netting set level, where the netting set can have several different types of deals and underlying driving risk-factors. Additionally, a CVA quote for a new deal, added or removed from an existing netting set, should be ideally priced in real-time. As the computational time for Monte Carlo simulations scales with number of scenarios, it is important that the standard error and bias of the results from the simulation are as small as possible. Variance reduction then is a highly desired feature for calculations related to CVA pricing.

An advantage of using SGBM is that it allows adapting the problem, to break the expectation, which would otherwise be solely computed using the Monte Carlo approach, to sub-problems where part of expectation is known in closed form. This feature helps in significantly bringing down the variance of the solution. In particular for the Bermudan swaptions, we employ hybrid measures to achieve variance reduction.

The $T$-forward measure, with corresponding expectation $\mathbb{E}^{T}$ and the $T$-maturity zero coupon bond $\boldsymbol{P}(\boldsymbol{t}, \boldsymbol{T})$ as the numeraire has the advantage that it allows for decoupling the payoff $\boldsymbol{V}(\boldsymbol{T})$ from the numeraire and take out the discount factor from the expectation, i.e.,

$$
\begin{aligned}
\boldsymbol{V}(\boldsymbol{t}) & =\boldsymbol{B}(\boldsymbol{t}) \mathbb{E}_{t}\left[\frac{\boldsymbol{V}(\boldsymbol{T})}{\boldsymbol{B}(\boldsymbol{T})}\right] \\
& =\boldsymbol{P}(\boldsymbol{t}, \boldsymbol{T}) \mathbb{E}_{t}^{T}[\boldsymbol{V}(\boldsymbol{T})] .
\end{aligned}
$$

One benefit, however, of the spot measure compared to the $T$-forward measure is that the numeraire asset $\boldsymbol{B}(\boldsymbol{t})$ is alive throughout the tenor and, therefore, allows for simulating paths irrespective of tenor. In SGBM, we will employ hybrid measures to obtain an efficient Monte Carlo simulation. In order to apply the hybrid measure we modify the inner-expectation, as given in Equation (12), to the following,

$$
Z_{n+1}=\mathbb{E}\left[\boldsymbol{V}_{n+1} \mid \zeta_{n+1}, \boldsymbol{r}_{\boldsymbol{n}}\right]
$$

which is followed by the computation of the following outer expectation (as opposed to Equation (13)),

$$
\mathrm{E}_{n}=\boldsymbol{B}\left(\boldsymbol{T}_{\boldsymbol{n}}\right) \mathbb{E}\left[\frac{\boldsymbol{Z}_{n+1}}{\boldsymbol{B}\left(\boldsymbol{T}_{n+1}\right)} \mid r_{n}\right] .
$$


The inner expectation is approximated by regression on short-rates simulated under the spot measure. Note that the minimization problem for regression problem changes from Equation (15) to:

$$
\min _{\alpha^{s}} \sum_{\boldsymbol{r}_{n}(\omega) \in \mathcal{B}_{n}^{s}}\left(\widehat{\boldsymbol{Z}}_{\boldsymbol{n}+1}^{s}(\omega)-\boldsymbol{V}_{\boldsymbol{n}+1}(\omega)\right)^{2} .
$$

The outer-expectation in Equation (18) can be computed under the $T$-forward measure, rather than the spot measure. This would allow computing the expectation, without explicitly simulating the bank account process $B$. The exposure at grid point $\boldsymbol{r}_{\boldsymbol{n}}(\omega)$ that belongs to bundle $\mathcal{B}_{\boldsymbol{n}}^{\boldsymbol{s}}$ is, therefore, computed as,

$$
\begin{aligned}
& \widehat{\mathrm{E}}_{n}(\omega)=\boldsymbol{B}\left(\boldsymbol{T}_{\boldsymbol{n}}, \omega\right) \mathbb{E}\left[\frac{\widehat{\boldsymbol{Z}}_{\boldsymbol{n}+1}^{s}}{\boldsymbol{B}\left(\boldsymbol{T}_{\boldsymbol{n}+1}\right)} \mid \boldsymbol{r}_{\boldsymbol{n}}(\omega)\right] \\
& =\boldsymbol{P}\left(\boldsymbol{T}_{n}, \boldsymbol{T}_{n+1}, \omega\right) \mathbb{E}^{T_{n+1}}\left[\hat{Z}_{n+1}^{s} \mid r_{n}(\omega)\right] \\
& \approx \boldsymbol{P}\left(\boldsymbol{T}_{n}, \boldsymbol{T}_{n+1}, \omega\right) \sum_{i=0}^{q} \alpha_{i, n+1}^{s} \mathbb{E}^{T_{n+1}}\left[\zeta_{i, n+1} \mid \boldsymbol{r}_{n}(\omega)\right]
\end{aligned}
$$

As $\zeta_{\boldsymbol{i}, \boldsymbol{n}+1}$ is a polynomial function of the short-rates (simulated in the Gaussian factor model), its conditional moments are known in closed form under the $T$ forward measure. An outcome of formulating the problem as above is that we only need to simulate the future option price, and not additionally the corresponding future bank account process, to obtain the option price on a given exposure date. As a result we achieve significant variance reduction in the exposure calculation when compared to plain LSM.

\subsubsection{The SGBM-CVA Algorithm}

As explained, SGBM computes the continuation value in two steps: we first compute the expected option value, conditioned on a finer information set, given by Equation (12), which is followed by the computation of the outer expectation, given by Equation (18).

The SGBM-CVA algorithm is, therefore, divided into two parts, a first and second pass. In the first pass, we perform a forward phase where $\boldsymbol{K}_{1}$ Monte Carlo paths are simulated, future cash flows are calculated and a regression basis is constructed. This is subsequently followed by a backward phase, where we estimate the payoffs and the polynomials by regression. In order to get unbiased values and lower bound values, we perform a second pass with a new forward phase where we simulate $\boldsymbol{K}_{2}$ Monte Carlo paths, evaluating the payoffs using the regression functions estimated in the first pass but with the new set of paths.

For clarity, we summarize the steps for the SGBM-CVA algorithm. 


\section{FIRST PASS: Estimate Regression Functions.}

(1) Generate $\boldsymbol{K}_{1}$ paths $\omega_{1}, \ldots, \omega_{\boldsymbol{K}_{1}}$, using Equation (2).

(2) For each path $\omega_{k}$ and time $\boldsymbol{T}_{\boldsymbol{n}}$, for $\boldsymbol{k}=1, \ldots, \boldsymbol{K}_{1}$ and $\boldsymbol{n}=1, \ldots, \boldsymbol{N}-1$, compute the state variable $\boldsymbol{r}_{\boldsymbol{n}}\left(\omega_{\boldsymbol{k}}\right)$ and values $\boldsymbol{V}_{\boldsymbol{n}}\left(\omega_{\boldsymbol{k}}\right)$, where $\boldsymbol{V}_{\boldsymbol{N}}$, is known and for $\boldsymbol{n}=$ $1, \ldots, N-1$, it is solved recursively as below.

(3) For each $\boldsymbol{n}=\boldsymbol{N}-1 \ldots, 1$,

(a) Bundle the grid points at $\boldsymbol{T}_{\boldsymbol{n}-1}$, into $a$ distinct bundles (except at $\boldsymbol{T}_{0}$, where there is only one point) using the algorithm described in Appendix B.

(b) Compute the regression functions, $Z_{n}^{s}, s=1, \ldots, a$, given by Equation (14), using the option values $V_{n}$ at $T_{n}$ for the paths originating from the $s$-th bundle, $\mathcal{B}_{n-1}^{s}$, at $\boldsymbol{T}_{n-1}$.

(c) Compute the $\mathrm{E}_{n}$ for the grid points in the $s$-th bundle at $\boldsymbol{T}_{\boldsymbol{n}-1}$, using Equation (20) for those paths for which $\boldsymbol{r}_{\boldsymbol{n}}\left(\omega_{\boldsymbol{k}}\right)$ belongs to the bundle $\mathcal{B}_{\boldsymbol{n}-1}^{s}$, for $s=1, \ldots, \boldsymbol{a}$.

\section{SECOND PASS: Estimate CVA.}

(1) In order to compute an unbiased CVA, generate a fresh set of $\boldsymbol{K}_{2}$ paths $\omega_{1}^{\prime}, \ldots, \omega_{\boldsymbol{K}_{2}}^{\prime}$, and compute new state variables $\boldsymbol{r}\left(\omega_{\boldsymbol{k}}^{\prime}\right)$ and values $\boldsymbol{V}_{\boldsymbol{n}}\left(\omega_{\boldsymbol{k}}^{\prime}\right)$

(2) For each $\boldsymbol{n}=\boldsymbol{N}-1 \ldots, 1$,

(a) Bundle the grid points at $\boldsymbol{T}_{\boldsymbol{n}-1}$ using the same algorithm as in the first pass and described in Appendix B.

(b) Compute the exposures for the grid points in bundle $s$, at time step $\boldsymbol{T}_{\boldsymbol{n}-1}$, using the regression function $Z_{n}^{s} s=1, \ldots, a$, obtained in the first pass.

(c) Compute the $\mathrm{EE}_{n}, \mathrm{EE}_{n}^{*}$ and $\operatorname{PFE}_{\alpha}\left(\boldsymbol{T}_{n}\right)$ for the grid points in the $s$-th bundle at $\boldsymbol{T}_{\boldsymbol{n}-1}$, for those paths for which $r_{n}\left(\omega_{k}^{\prime}\right)$ belongs to the bundle $\mathcal{B}_{\boldsymbol{n}-1}^{s}$, for $\boldsymbol{s}=1, \ldots, \boldsymbol{a}$.

(3) The CVA charge is then calculated as,

$$
\mathrm{CVA} \approx\left(1-\boldsymbol{R}_{\boldsymbol{C}}\right) \sum_{\boldsymbol{n}=0}^{N-1} \mathrm{EE}_{\boldsymbol{n}}^{*}\left(\boldsymbol{P} \boldsymbol{D}_{\boldsymbol{n}+1}-\boldsymbol{P} \boldsymbol{D}_{\boldsymbol{n}}\right)
$$

Remark 3.2 Valuation of Bermudan swaptions with American Monte Carlo methods requires an estimate of the early exercise boundary. Exposure can then be seen as a barrier option (knock-in) with the estimated exercise boundary as the barrier. Once the option has been exercised (knocked) along a path at time $\boldsymbol{T}_{\boldsymbol{n}}$ the exposure $E_{m}$ at $\boldsymbol{T}_{\boldsymbol{m}}$ for $\boldsymbol{T}_{\boldsymbol{n}}<\boldsymbol{T}_{\boldsymbol{m}}$ for that path becomes zero.

Remark 3.3 The market standards for swaptions are cash-settled contracts, i.e., contracts that settle into a cash payment when exercised. The benefit is that one avoids credit exposure (and the obligation of collateral posting due to the Credit Support Annex, or CSA) and therefore have zero exposure after the exercise date. For physically settled contracts, i.e., contracts entered into an interest rate swap when the contract is exercised, one would have to calculate the exposure of the swap from the exercise date throughout the swap tenor. The Bermudan swaption formulas in Section 2.2 describe physical-settled contracts. The standard pricing formulas for cash-settled agreements are not properly 
justified, since one would have to calculate the annuity $\boldsymbol{A}_{n, m}$ by discounting at the fixed swap rate $\boldsymbol{S}_{\boldsymbol{n}, \boldsymbol{m}}\left(\boldsymbol{T}_{0}\right)$. Since the SGBM-CVA algorithm presented here works irrespectively of settlement type, we assume for simplicity that the annuity for cash-and physical-settled Bermudan swaptions are the same.

\section{Numerical Results}

In this section, we study the performance of SGBM-CVA by means of numerical experiments. The numerical examples presented below demonstrate the efficiency of calculating CVA using SGBM.

\subsection{Setup}

We use a one-dimensional market state variable $\boldsymbol{r}(\boldsymbol{t})$ to represent the market information, and we let the short rates be simulated using the HW1F model by Hull and White (1990), which is commonly used for pricing Bermudan swaptions. Under HW1F, the short rate dynamics are given by Equation (1) with $\kappa$ and $\sigma$ constant. We calibrate the HW1F model parameters to the initial zero coupon bond prices observed in the market 2 January 2014. For the default probability function in equation (7), we set the hazard rate $\gamma(\boldsymbol{t})=0.05$, and the recovery rate $\boldsymbol{R}_{\boldsymbol{C}}=0.40$.

For the LSM and SGBM regression, we use a third-order polynomial with the short rate as the basis and $\zeta_{i, n}=r_{n}^{i}$. The moments for the short rates under the HW1F dynamics in Equation (1) are given in Appendix A.

We consider Bermudan swaptions exercisable once a year with Moneyness ( $\mathrm{MN})$ i.e., the spot vs. strike ratio of $80 \%, 100 \%$ and $120 \%$, and with realistic HW1F parameters $\kappa=0.01,0.02$ and $\sigma=0.01,0.02 .^{3}$

We simulate the first pass with $\boldsymbol{K}_{1}=4096$ seeds using the Mersenne twister pseudo random number generator to estimate the regression functions. Subsequently, we simulate the second pass with $\boldsymbol{K}_{2}=8192$ quasi-Monte Carlo Sobol paths using the regression function estimated in the first pass to estimate the unbiased Bermudan swaptions values, EE, PFE and CVA charges. Each test is repeated 100 times with different seeds in the first pass, to remove the overall influence of the Mersenne twister pseudo random number generator.

We use the bundling scheme described in Appendix B with 8 bundles and with the same number of bundles at each time step, except at time $\boldsymbol{T}_{0}$, where there is only one point, $\boldsymbol{r}_{0}$. We report the values obtained from the second pass. The prices are reported in basis points, with the notional $\mathcal{N}=10,000$.

The variance reduction is defined as the ratio between the variance from LSM and the variance from SGBM, where both estimates are obtained from 100 simulations.

\subsection{EE and PFE Values}

Figure 3 illustrates the $\mathrm{PFE}_{5 \%}, \mathrm{PFE}_{95 \%}$ and $\mathrm{EE}$ values generated by LSM and SGBM for $5 \mathrm{Y}, 10 \mathrm{Y}, 15 \mathrm{Y}$ and $20 \mathrm{Y}$ Bermudan swaptions. We observe that both methods generate 

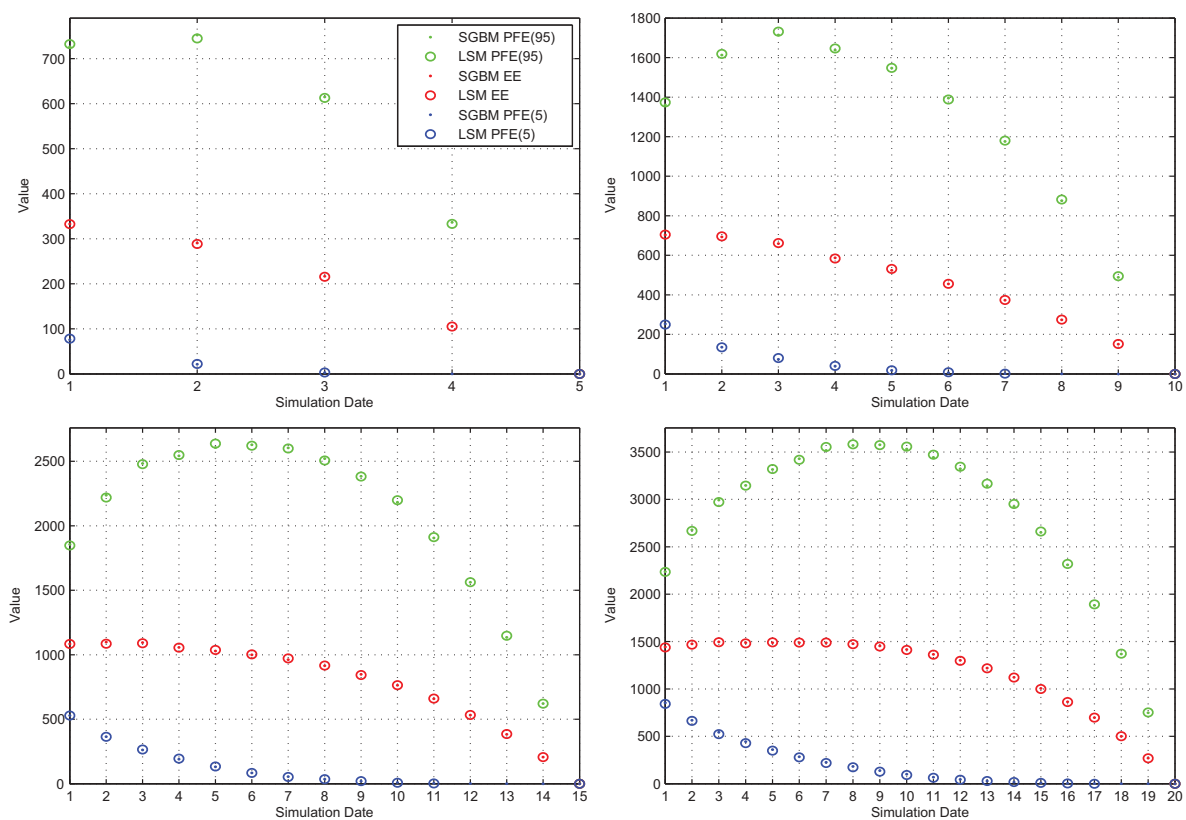

Figure 3. Bermudan swaption $\mathrm{EE}_{1} \mathrm{PFE}_{5 \%}$ and $\mathrm{PFE}_{95 \%}$ under $\mathrm{HW} 1 \mathrm{~F}$ with $\kappa=0.01$ and $\sigma=0.01$ with notional $\mathcal{N}=10,000$. Upper Left: $5 Y$ Maturity. Upper Right: 10Y Maturity. Lower Left: $15 Y$ Maturity. Lower Right: 20Y Maturity.

the same values and the characteristic shapes, i.e., the exposure tends to increase first, since there is an increased probability that the Bermudan swaptions will be deeper inthe-money at a future exercise date.

The efficiency of SGBM compared to LSM for estimating $\mathrm{PFE}_{5 \%}, \mathrm{PFE}_{95 \%}$ and $\mathrm{EE}$ is illustrated in Figure 4. Clearly, LSM is affected by outliers for the high quantile PFE estimation. For the EE by SGBM, we obtain on average a variance reduction of a factor 100 .

\subsection{CVA}

For the CVA computations, we consider Bermudan swaptions with maturities of 5Y and $10 \mathrm{Y}$. Tables 1 and 2 report the lower bound values for the Bermudan swaptions and CVA charges via LSM and SGBM. The numbers in parentheses are sample standard deviations and the values from LSM and SGBM differ at most 5 bps. As a first observation, the standard deviation for the SGBM lower bounds is much smaller than the ones obtained from LSM. The efficiency of SGBM compared to LSM for pricing and CVA calculation is illustrated in Figure 5. For the lower volatility scenarios, i.e., $\sigma=$ 0.01 we obtain for the $5 Y$ Bermudan swaption CVA a variance reduction of a factor 200 and for the $10 \mathrm{Y}$ a factor of 400 . For the high volatility case, i.e., with $\sigma=0.02$ we observe a variance reduction of a factor 100 for the $5 \mathrm{Y}$ and 200 for the $10 \mathrm{Y}$ test case. The interpretation here is that for a 10Y Bermudan swaption under HW1F with $\sigma=$ 0.01 we will on average need 400 times more Monte Carlo seeds for LSM compared to SGBM in order to obtain equally 'accurate' CVA values. 

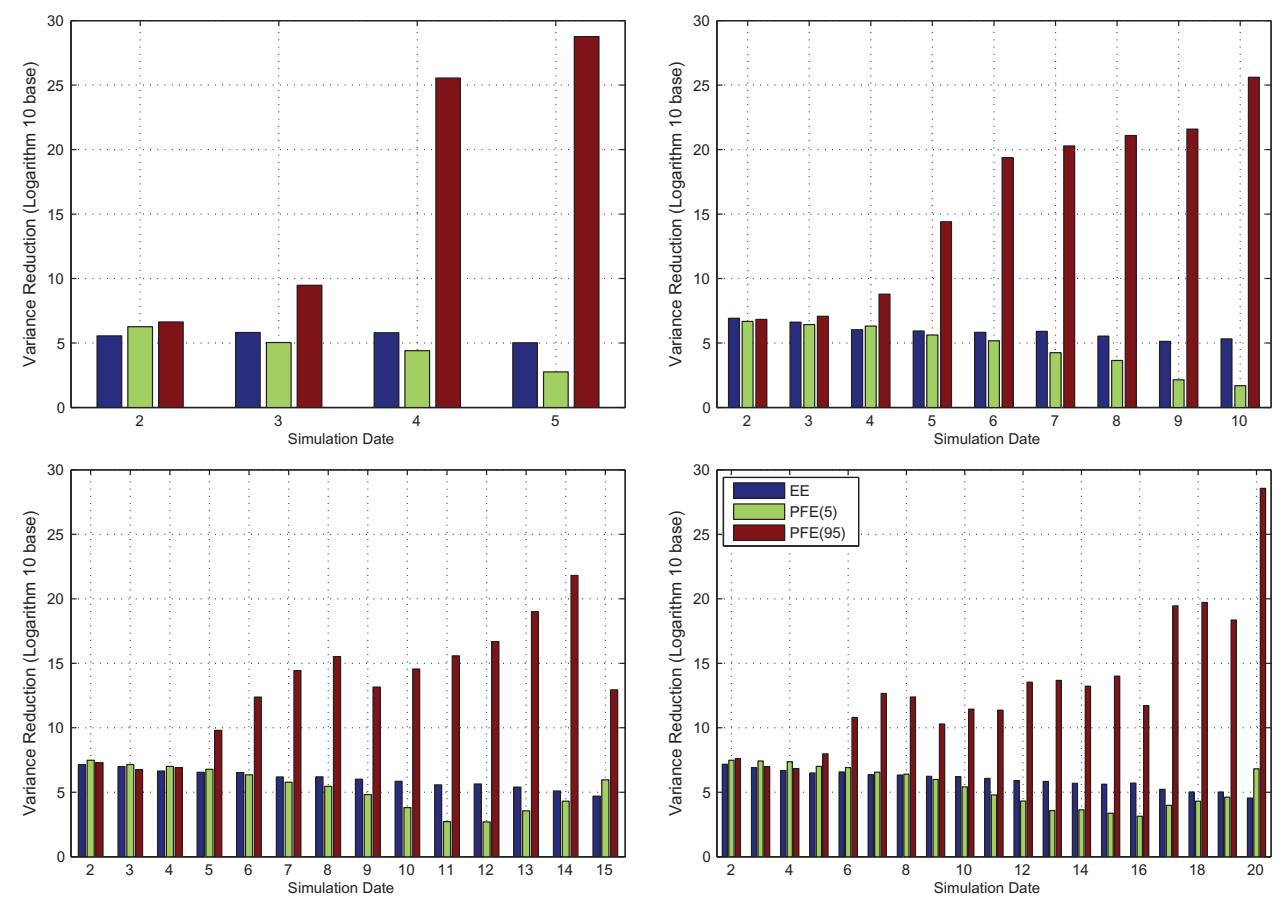

Figure 4. $\mathrm{EE}, \mathrm{PFE}_{5 \%}$ and $\mathrm{PFE}_{95 \%}$ variance reduction for a Bermudan swaption under HW1F with $\kappa=0.01, \sigma=0.01$ and notional $\mathcal{N}=10,000$. Upper Left: $5 Y$ Maturity. Upper Right: 10Y Maturity. Lower Left: 15Y Maturity. Lower Right: 20Y Maturity.

Table 1. Lower bound 5Y Bermudan swaption risk-free prices and CVA charges under HW1F using LSM and SGBM. Prices are in basis points and standard deviations within parentheses.

\begin{tabular}{ccccccccccc}
\hline MN & $\kappa$ & $\sigma$ & \multicolumn{2}{c}{ SGBM } & \multicolumn{2}{c}{ LSM } & \multicolumn{2}{c}{ CVA SGBM } & \multicolumn{2}{c}{ CVA LSM } \\
\hline 0.8 & 0.01 & 0.01 & 477.30 & $(0.1732)$ & 477.39 & $(3.8995)$ & 51.70 & $(0.0144)$ & 51.70 & $(0.2105)$ \\
1.0 & 0.01 & 0.01 & 548.12 & $(0.1827)$ & 548.26 & $(4.1316)$ & 58.58 & $(0.0140)$ & 58.53 & $(0.2206)$ \\
1.2 & 0.01 & 0.01 & 599.25 & $(0.1824)$ & 599.65 & $(4.3217)$ & 63.41 & $(0.0141)$ & 63.35 & $(0.2140)$ \\
0.8 & 0.01 & 0.02 & 736.43 & $(0.4557)$ & 737.74 & $(7.0878)$ & 76.31 & $(0.0347)$ & 75.41 & $(0.3590)$ \\
1.0 & 0.01 & 0.02 & 801.25 & $(0.4585)$ & 803.21 & $(7.1382)$ & 82.29 & $(0.0327)$ & 83.04 & $(0.3801)$ \\
1.2 & 0.01 & 0.02 & 846.78 & $(0.4973)$ & 847.85 & $(7.3205)$ & 86.45 & $(0.0337)$ & 86.16 & $(0.3525)$ \\
0.8 & 0.02 & 0.01 & 471.07 & $(0.1776)$ & 471.89 & $(4.1821)$ & 51.06 & $(0.0149)$ & 51.32 & $(0.2222)$ \\
1.0 & 0.02 & 0.01 & 542.15 & $(0.1792)$ & 542.76 & $(4.0907)$ & 57.99 & $(0.0140)$ & 58.01 & $(0.2165)$ \\
1.2 & 0.02 & 0.01 & 593.49 & $(0.1783)$ & 594.28 & $(4.5220)$ & 62.86 & $(0.0132)$ & 63.06 & $(0.2133)$ \\
0.8 & 0.02 & 0.02 & 723.13 & $(0.4520)$ & 726.45 & $(6.898)$ & 75.03 & $(0.0347)$ & 74.86 & $(0.3722)$ \\
1.0 & 0.02 & 0.02 & 788.16 & $(0.4595)$ & 791.75 & $(6.8509)$ & 80.96 & $(0.0343)$ & 80.88 & $(0.3517)$ \\
1.2 & 0.02 & 0.02 & 833.95 & $(0.4567)$ & 837.23 & $(6.9155)$ & 85.13 & $(0.0324)$ & 85.63 & $(0.3872)$ \\
\hline
\end{tabular}

\subsection{Approximation Error}

For the approximation error we study the convergence by increasing the number of paths in the first and the second pass. As the 'true' value, we select the mean of the LSM computations with $K_{1}=131,072$ and $K_{2}=2 K_{1}$, repeated 100 times. Then, for different values of $\boldsymbol{K}_{1}$, with $\boldsymbol{K}_{2}=2 \boldsymbol{K}_{1}$, we repeat the simulation 100 times, and estimate the relative error with respect to the 'true' value, for LSM and SGBM with 1, 2, 4, 8 and 16 bundles. In Figure 6, we illustrate the mean and the standard deviation of the error for a $5 \mathrm{Y}$ Bermudan swaption with $\kappa=0.01, \sigma=0.01$ and an 
Table 2. Lower bound 10Y Bermudan swaption risk-free prices and CVA charges under HW1F using LSM and SGBM. Prices are in basis points and standard deviations within parentheses.

\begin{tabular}{ccccccccccc}
\hline MN & $\kappa$ & $\sigma$ & \multicolumn{2}{c}{ SGBM } & \multicolumn{2}{c}{ LSM } & \multicolumn{2}{c}{ CVA SGBM } & \multicolumn{2}{c}{ CVA LSM } \\
\hline 0.8 & 0.01 & 0.01 & 947.30 & $(0.2326)$ & 946.74 & $(7.8717)$ & 175.04 & $(0.0340)$ & 175.41 & $(0.6631)$ \\
1.0 & 0.01 & 0.01 & 1187.0 & $(0.2355)$ & 1186.7 & $(8.7668)$ & 215.55 & $(0.0346)$ & 214.56 & $(0.7524)$ \\
1.2 & 0.01 & 0.01 & 1367.7 & $(0.2259)$ & 1368.2 & $(9.0424)$ & 245.44 & $(0.0327)$ & 244.85 & $(0.6960)$ \\
0.8 & 0.01 & 0.02 & 1584.1 & $(0.5393)$ & 1586.4 & $(13.252)$ & 283.59 & $(0.0747)$ & 282.52 & $(1.1481)$ \\
1.0 & 0.01 & 0.02 & 1805.5 & $(0.5513)$ & 1809.4 & $(13.588)$ & 319.16 & $(0.0760)$ & 319.46 & $(1.1454)$ \\
1.2 & 0.01 & 0.02 & 1966.0 & $(0.5317)$ & 1968.5 & $(14.517)$ & 344.73 & $(0.0691)$ & 343.11 & $(1.1505)$ \\
0.8 & 0.02 & 0.01 & 921.21 & $(0.2283)$ & 920.38 & $(8.2133)$ & 170.12 & $(0.0337)$ & 170.34 & $(0.7274)$ \\
1.0 & 0.02 & 0.01 & 1162.7 & $(0.2170)$ & 1161.2 & $(8.5945)$ & 211.08 & $(0.0324)$ & 210.45 & $(0.7198)$ \\
1.2 & 0.02 & 0.01 & 1345.0 & $(0.2367)$ & 1344.3 & $(8.8313)$ & 241.23 & $(0.0344)$ & 241.07 & $(0.7121)$ \\
0.8 & 0.02 & 0.02 & 1529.5 & $(0.5057)$ & 1536.7 & $(12.958)$ & 273.61 & $(0.0717)$ & 274.5 & $(1.1336)$ \\
1.0 & 0.02 & 0.02 & 1752.3 & $(0.5428)$ & 1758.7 & $(14.181)$ & 309.44 & $(0.0763)$ & 311.73 & $(1.1552)$ \\
1.2 & 0.02 & 0.02 & 1914.2 & $(0.5630)$ & 1919.6 & $(13.726)$ & 335.13 & $(0.0729)$ & 334.72 & $(1.1953)$ \\
\hline
\end{tabular}
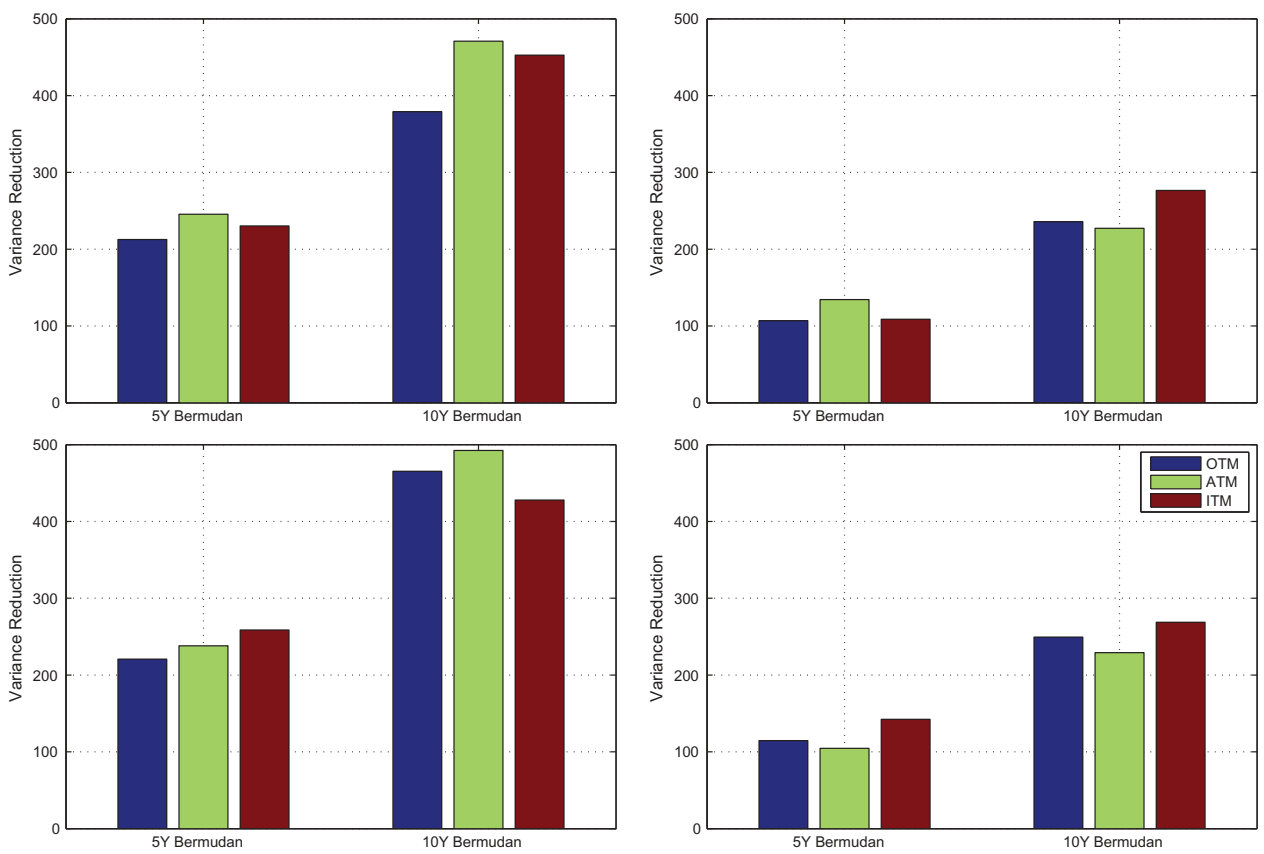

Figure 5. CVA variance reduction for $5 \mathrm{Y}$ and 10Y Bermudan swaptions under HW1F with notional $\mathcal{N}=10,000$. Upper Left: $k=0.01, \sigma=0.01$. Upper Right: $\kappa=0.01 \sigma=0.0 .2$. Lower Left: $\kappa=0.02$ $\sigma=0.01$. Lower Right: $\kappa=0.02, \sigma=0.02$.

MN value of $100 \%$. One can observe that LSM requires a large number of paths to converge to the true value. For SGBM-1 (i.e. SGBM with 1 bundle) we see an upward-biased value, but we observe a significant improvement in convergence by SGBM-2 which converges at $\boldsymbol{K}_{1}=4096$ demonstrating essentially the same accuracy as LSM in the case of $K_{1}=131,072$ paths. The error is further reduced by increasing the number of bundles and the computations with 4,8 and 16 bundles converge at $K_{1}=16384$ paths. SGBM-16 is slightly upward-biased for small numbers of paths, most likely because some bundles will then contain too few paths to allow a feasible regression without too large error. It can be seen that the SGBM-16 is slightly 

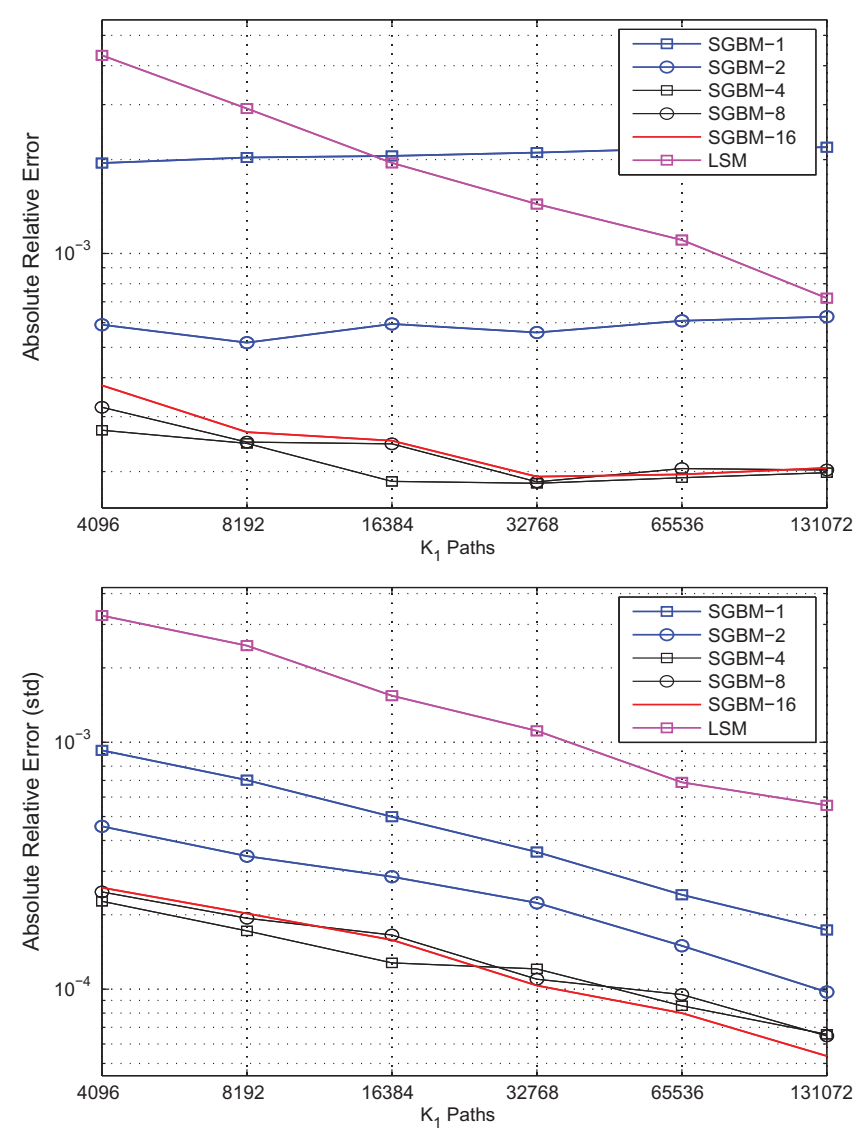

Figure 6. CVA error. Mean and standard deviation for 5Y Bermudan swaption.

upward-bias for low number of paths compared to SGBM-4 and SGBM-8. We observe similar patterns and convergence for different $\mathrm{MN}$ values, maturities and parameter setup, when the number of paths per bundle is too small.

SGBM demonstrate a faster convergence and produces more stable values with significant lower variances. The reason is that LSM uses the regressed continuation values directly to make the early-exercise decision. A large number of paths and basis functions are required to reduce the noise in this regressed function. Therefore, the quality of the LSM early-exercise policy may not be accurate for a small number of paths and basis functions. In SGBM, however, the regressed function is just the inner expectation, which is not used for decision-making. The outer expectation, which can be analytically computed, gives the continuation value and is used to make the early exercise decision. As the noise, or the error due to regression, is normally distributed with a zero mean, the outer expectation of the noise would be zero.

\section{Conclusion}

Usually banks have a large number of trades in a portfolio and it would be computationally inefficient to require several runs for the trades in the portfolio to get a CVA 
which we can be confident about if there is high variance. This paper presented the application of the Stochastic Grid Bundling Method (SGBM) for calculating exposures, potential future exposure and approximating CVA charges for Bermudan swaptions in an American Monte Carlo simulation framework. SGBM is a regression-based Monte Carlo method which is accurate and easy to implement. Variance reduction, based on iterated conditioning, in combination with the bundling technique forms the necessary ingredients for accurate CVA valuation with a relative small number of paths and basis functions. The computational time for the method is comparable to the least squares method in Longstaff and Schwartz (2001), but a higher accuracy is achieved. Our numerical examples demonstrate the efficiency of calculating CVA using SGBM, making it a very suitable candidate with a potential to calculate 'real-time' CVA charges and easy extension to other charges within the XVA family.

\section{A HW1F Moments}

Let $\boldsymbol{M}_{\boldsymbol{k}}(\boldsymbol{s}, \boldsymbol{t})=\mathbb{E}\left[\boldsymbol{r}(\boldsymbol{t})^{\boldsymbol{k}} \mid \boldsymbol{s}\right]$ be the $k$-th moment. The three first moments for the HW1F are given by,

$$
\begin{gathered}
\boldsymbol{M}_{1}(\boldsymbol{s}, \boldsymbol{t})=\boldsymbol{e}^{-\kappa(\boldsymbol{t}-\boldsymbol{s})} \boldsymbol{r}(\boldsymbol{s})+\frac{\theta(\boldsymbol{t})}{\kappa}\left(1-\boldsymbol{e}^{-\kappa(t-s)}\right), \\
\boldsymbol{M}_{2}(\boldsymbol{s}, \boldsymbol{t})=\boldsymbol{M}_{1}^{2}(\boldsymbol{s}, \boldsymbol{t})+\frac{\sigma^{2}}{2 \kappa}\left(1-\boldsymbol{e}^{-2 \kappa(t-s)}\right), \\
\boldsymbol{M}_{3}(\boldsymbol{s}, \boldsymbol{t})=\boldsymbol{M}_{1}^{3}(\boldsymbol{s}, \boldsymbol{t})+3 \boldsymbol{M}_{1}(\boldsymbol{s}, \boldsymbol{t})\left(\boldsymbol{M}_{2}(\boldsymbol{s}, \boldsymbol{t})-\boldsymbol{M}_{1}^{2}(\boldsymbol{s}, \boldsymbol{t})\right) .
\end{gathered}
$$

\section{B Bundling}

The goal is to construct bundles by generating $K$ replications of the underlying asset path, $\boldsymbol{r}_{\boldsymbol{n}}\left(\omega_{\boldsymbol{k}}\right)$, for $\boldsymbol{n}=1, \ldots, \boldsymbol{N}$ and $\boldsymbol{k}=1, \ldots, \boldsymbol{K}$ and to bundle them at each epoch, $\boldsymbol{T}_{\boldsymbol{n}}$, into $\boldsymbol{a}_{\boldsymbol{n}}(\boldsymbol{K})$ non-overlapping sets, $\mathcal{B}_{n}^{s}=\left(\mathcal{B}_{n}^{1}, \ldots, \mathcal{B}_{n}^{a}\right)$, with a threshold, $\boldsymbol{b}_{\boldsymbol{n}}(\boldsymbol{K})$, on the number of path points in each bundle. This is done by defining at each epoch, $\boldsymbol{T}_{n}$, representative states $\mu_{n}^{s}$ for $s=1, \ldots, \boldsymbol{a}_{n}(\boldsymbol{K})$. The $s$-th bundle at time $\boldsymbol{T}_{\boldsymbol{n}}$ is thus defined as

$$
\mathcal{B}_{n}^{s}=\left\{\boldsymbol{r}_{n}\left(\omega_{k}\right):\left\|\boldsymbol{r}_{n}\left(\omega_{k}\right)-\mu_{n}^{s}\right\|_{2} \leq\left\|\boldsymbol{r}_{n}\left(\omega_{k}\right)-\mu_{n}^{\ell}\right\|_{2} \forall 1 \leq \ell \leq \boldsymbol{a}_{\boldsymbol{n}}(\boldsymbol{K})\right\},
$$

for $\boldsymbol{k}=1, \ldots, \boldsymbol{K}$ and where $\mu_{n}^{s}$ is the mean of the points in $\mathcal{B}_{n}^{s}$.

Suppose we need to bundle $\boldsymbol{K}_{\boldsymbol{s}}$ grid points at epoch $\boldsymbol{T}_{\boldsymbol{n}}$, given by $\boldsymbol{r}_{\boldsymbol{n}}\left(\omega_{\boldsymbol{k}}\right)$, where $\boldsymbol{k}=$ $1, \ldots, \boldsymbol{K}_{\boldsymbol{s}}$. The following steps are performed recursively.

(1) Compute the mean of the given set of grid points,

$$
\mu_{n}^{s}=\frac{1}{K_{s}} \sum_{k=1}^{K_{s}} r_{n}\left(\omega_{k}\right)
$$

(2) Bundling of the grid points is performed by dividing the grid points into two groups, depending on whether the asset price for the grid point is greater than or less than the mean of the asset prices for the given set of grid points: 


$$
\begin{gathered}
\mathcal{B}_{n}^{1}\left(\omega_{k}\right)=\mathbf{I}\left(\boldsymbol{r}_{n}\left(\omega_{k}\right)>\mu_{n}^{s}\right), \\
\mathcal{B}_{n}^{2}\left(\omega_{k}\right)=\boldsymbol{I}\left(\boldsymbol{r}_{n}\left(\omega_{k}\right) \leq \mu_{n}^{s}\right),
\end{gathered}
$$

for $\boldsymbol{k}=1, \ldots, \boldsymbol{K}_{\boldsymbol{s}} \cdot \mathcal{B}_{n}^{1}\left(\omega_{\boldsymbol{k}}\right)$ returns 'true', when the state variable $\boldsymbol{r}_{\boldsymbol{n}}\left(\omega_{\boldsymbol{k}}\right)$ is greater than the mean, $\mu_{n}^{s}$ and belongs to bundle $1 . \mathcal{B}_{n}^{2}\left(\omega_{k}\right)$ returns 'true', if it less than the mean and belongs to bundle 2 . Formally, $\mathcal{B}_{n}^{s}\left(\omega_{k}\right)$ returns 'true', if the grid point $\boldsymbol{r}_{\boldsymbol{n}}\left(\omega_{\boldsymbol{k}}\right)$ belongs to bundle $s$.

(3) Bundles $\mathcal{B}_{n}^{1}$ and $\mathcal{B}_{n}^{2}$ can be split again, returning to step 1.

\section{Notes}

1. Basel III states that 'Whenever such a CDS spread is not available, the bank must use a proxy spread that is appropriately based on the rating, industry and region of the counterparty'. Calibration methods ranked from best to worst, first, from CDS spreads (if traded and quoted in the marked), second, from bond spreads (if traded and quoted in the marked), and third, from a rating transition matrix and last, from proxies such as stock prices or reported fundamental data.

2. The International Swaps and Derivatives Association (ISDA) defines the wrong-way risk as 'the risk that occurs when exposure to a counterparty is adversely correlated with the credit quality of that counterparty'. If these two effects tend to happen together, then that co-dependence will increase the CVA on the contract and it will make the CVA larger than when the effects were independent. For details on WWR see for instance Hull and White (2012), Rosen and Saunders (2012), Redon (2006)

3. For instance, at the beginning of 2015 , the HW1F, with value of $\kappa$ and $\sigma$ calibrated to USD, co-terminal swaptions were both around 0.01 .

\section{References}

Andersen, L. 2000. "A Simple Approach to the Pricing of Bermudan Swaptions in the MultiFactor LIBOR Market Model.” The Journal of Computational Finance 3: 5-32. doi:10.21314/ JCF.1999.041.

Andersen, L., and M. Broadie. 2004. "A Primal-Dual Simulation Algorithm for Pricing MultiDimensional American Options." Management Science 50: 1222-1234. doi:10.1287/ mnsc.1040.0258.

Belomestny, D., C. Bender, and J. Schoenmakers. 2009. "True Upper Bounds for Bermudan Products via Non-Nested Monte Carlo." Mathematical Finance 19: 53-71. doi:10.1111/ mafi.2008.19.issue-1.

Bender, C., and J. Schoenmakers. 2006. "An Iterative Method for Multiple Stopping: Convergence and Stability." Advances in Applied Probability 38: 729-749. doi:10.1017/ S0001867800001245.

Brigo, D., and A. Pallavicini. 2013. Counterparty Credit Risk, Collateral and Funding: With Pricing Cases For All Asset Classes. Chichester: Wiley.

Brigo, D., and F. Mercurio. 2001. Interest Rate Models: Theory and Practice - with Smile, Inflation and Credit. 2nd Ed. Heidelberg: Springer Verlag.

Broadie, M., and P. Glasserman. 2004. "A Stochastic Mesh Method for Pricing HighDimensional American Options." The Journal of Computational Finance 7: 35-72. doi:10.21314/JCF.2004.117. 
Canabarro, E., and D. Duffie. 2003. "Measuring and Marking Counterparty Risk." Chap. 9 in Asset/Liability Management of Financial Institutions, edited by L. Tilman, 122-134. New York: Institutional Investor Books.

Carriere, J. 1996. "Valuation of Early-Exercise Price of Options Using Simulations and Nonparametric Regression." Insurance Mathematics and Economics 19: 19-30.

Glasserman, P., and B. Yu. 2004. "Number of Paths versus Number of Basis Functions in American Option Pricing." Annals of Applied Probability 14: 2090-2119.

Gregory, J. 2010. Counterparty Credit Risk and Credit Value Adjustment: A Continuing Challenge for Global Financial Markets. Chichester: Wiley.

Haugh, M., and L. Kogan. 2004. "Pricing American Options: A Duality Approach." Operations Research 52: 258-270. doi:10.1287/opre.1030.0070.

Hull, J., and A. White. 1990. "Pricing Interest-Rate Derivative Securities." The Review of Financial Studies 3: 573-592. doi:10.1093/rfs/3.4.573.

Hull, J., and A. White. 2012. "CVA and Wrong Way Risk." Financial Analysts Journal 68: 58-69. doi:10.2469/faj.v68.n5.6.

Jain, S., and C. W. Oosterlee. 2015. "The Stochastic Grid Bundling Method: Efficient Pricing of Bermudan Options and Their Greeks." Applied Mathematics and Computation 269: 412-431. doi:10.1016/j.amc.2015.07.085.

Jin, X., H. H. Tana, and J. Sun. 2007. "A State-Space Partitioning Method for Pricing HighDimensional American-Style Options.” Mathematical Finance 17: 399-426. doi:10.1111/ mafi.2007.17.issue-3.

Kolodko, A., and J. Schoenmakers. 2004. "Upper Bounds for Bermudan Style Derivatives." Monte Carlo Methods and Applications 10: 331-343. doi:10.1515/mcma.2004.10.3-4.331.

Kolodko, A., and J. Schoenmakers. 2006. "Iterative Construction of the Optimal Bermudan Stopping Time." Finance and Stochastics 10: 27-49. doi:10.1007/s00780-005-0168-5.

Longstaff, F. A., and E. S. Schwartz. 2001. "Valuing American Options by Simulation: A Simple Least-Squares Approach." Review of Financial Studies 14: 113-147. doi:10.1093/rfs/14.1.113.

Picoult, E. 2005. Calculating and Hedging Exposure, Credit Value Adjustment and Economic Capital for Counterparty Credit Risk in Counterparty Credit Risk Modelling. London: Risk Books.

Piterbarg, V. 2004. "Pricing and Hedging Callable Libor Exotics in Forward Libor Models." The Journal of Computational Finance 8: 65-117. doi:10.21314/JCF.2005.140.

De Prisco, B., and D. Rosen. 2005. "Modeling Stochastic Counterparty Credit Exposures for Derivatives Portfolios." In Counterparty Credit Risk Modeling, edited by M. Pykhtin. London: Risk Books.

Pykhtin, M., and D. Rosen. 2010. "Pricing Counterparty Risk At The Trade Level and Credit Valuation Adjustment Allocations." The Journal of Credit Risk 6: 3-38. doi:10.21314/ JCR.2010.116.

Pykhtin, M., and S. Zhu. 2007. "A Guide to Modeling Counterparty Credit Risk.” GARP Risk Review 37: 16-22.

Redon, C. 2006. “Wrong Way Risk Modelling.” Risk Magazine, April, 90-95.

Rogers, L. 2002. "Monte Carlo Valuation of American Options." Mathematical Finance 12: 271286. doi:10.1111/mafi.2002.12.issue-3.

Rosen, D., and D. Saunders. 2012. "CVA the Wrong Way." Journal of Risk Management in Financial Institutions 5: 252-272.

Tilley, J. A. 1993. "Valuing American Options in a Path Simulation Model." Transactions of the Society of Actuaries 45: 83-104. 